\title{
Design and Implementation of British and American Literature Learning Platform based on Android Mobile Systems
}

\author{
Yanyan LEI ${ }^{1, a}$ \\ ${ }^{1}$ Hechi University College of Foreign Languages, Yizhou 546300,China \\ ayanyanlei@126.com
}

\begin{abstract}
Keywords: Online Learning Platform; British and American Literature; Android System; Mobile Devices; Multi-user Organization.
\end{abstract}

\begin{abstract}
With the bursting development of computer science and mobile devices, the combination of education and recent techniques is urgently needed. In view of the existing mobile learning system interactive poor, renewable resources, shortcomings and so on big data traffic, this paper puts forward a kind of mobile learning system based on Android platform. The system can not only solve the above problem, also can easily implement integration and extension. With the rapid popularization of smart mobile terminals, mobile study become a hot spot, the trend of traditional live remote education system puts forward a new challenge. Therefore, in this paper, we undertake research on British and American literature learning platform based on Android mobile systems. We modify the framework of the current Android system and re-design the user interface. The simulation result shows the system is user-friendly and stable.
\end{abstract}

\section{Introduction}

Smart phones, PDAS, tablets, and the popularity of other portable communication equipment makes mobile learning to be a new way of learning. In view of the existing mobile learning system interactive poor, renewable resources, shortcomings and so on big data traffic, this paper puts forward a kind of mobile learning system based on Android platform. The system can not only solve the above problem, also can easily implement integration and extension. With the rapid popularization of smart mobile terminals, mobile study become a hot spot, the trend of traditional live remote education system puts forward a new challenge [1-2]. Often bandwidth in the network of mobile learning environment is low and can't guarantee the reliable and durable connection, users prone to temporarily lost in the process of mobile data network coverage, or when they switch between 3G and wifi connection is broken, the traditional streaming media based on the stable network protocol no longer apply [3-4]. On the other hand, with the increasingly rich Internet resources, the teacher teaching means are also increasingly diversified. Teachers in teaching, for example, to insert a demo video broadcast to assist teaching or lectures, and such traditional slide show scenes and video playback scenarios of hybrid coding in the demo scene, usually because of the screen of the computer screen resolution is higher or the traditional coding techniques of low output frame rate lead to screen smooth video playback is not enough; And general video coding techniques for screen contents of natural images and text edge part adopts the indiscriminate quantitative processing, decoding the text image after the vague and often with visible traces of artificial processing, unable to meet the requirements of customers for a static image quality. In the SMS technology of mobile learning system, mobile phone and server via SMS to complete information exchange, learning resources to text, MMS can be achieved by mixing [5]. This system main advantage is information feedback in time; Faults mainly poor interactivity, construction operation maintenance cost is higher. Special for mobile learning network conditions using HTTP-Streaming technology in the live system. Will move course of audio, video and screen video stream on the server side according to certain time interval segment and save the file, the client only need to use the HTTP protocol can sequentially got all the data from the server. If because the network environment in the process of transmission of changes in connection is broken, need to restart the HTTP request [6-8]. 
Mobile learning is in information technology and related to mobile devices, with the help of learners at any given time and place of learning, is a kind of casual, informal learning activities. Mobile learning is a kind of new learning style, it uses the mobile communication device must be able to effectively present learning content and support two-way communication between teachers and learners. It is mobile communication technology, Internet technology and the combination of remote education, can at anytime, anywhere, with you to learn, is further extension of distance education under the condition of new technology and development. Smart phone as a mobile terminal, it is a smart phone enthusiasts affectionately called "a little computer," has made mobile learning anytime and anywhere, so the advantage of using smart phones for mobile learning is very clear. (1) Break the limit of time and space, the largest within the scope of use of information. Mobile learning is not restricted by geographical space and the study way, the mobile terminal portable degree decides the learners learning convenience; (2) Provide technical support, making the mobile learning more active. Mobile learning resources are present in the form of multimedia, and a variety of media formats the intelligent mobile phone technology development make mobile learning has been the biggest technical support; (3) The realization of individualized learning. Learners will be because of the difference of regional culture has the very big difference in learning styles, they are learning problems in very different also, collective teaching, however, it is hard to meet such requirements; (4) Timeliness is strong. Learners may encounter all kinds of problems in the learning process, because of the limitations, they cannot solve their problems, you can use the QQ in the smartphone communications software through the network to ask the teacher questions, quickly solve the problem was, as well as to the grasp of knowledge.

In this research article, we conduct research on the British and American literature learning platform based on android mobile systems. We firstly analyze the traditional structure of Android platform with theoretical analysis and application implementations. Later, we design the proposed platform with user interface design. Finally, the experiment is conducted.

\section{Design and Implementation of the Learning System}

The Concepts of Android System. Smartphone application is booming in the process of teaching, for mobile learning opportunity has been brought by lots of people in the education industry are discovered and put into action, it not only broke through the regional restrictions, and more on technology is more suitable for the need of mobile learning. As people life rhythm speeding up, needs a more flexible approach to learning, as the city expanding, people in the spare time on the road more and more long, smart phone study will enable students of various ages to the concept of learning content through smart phones, make full use of limited time to study. Mobile learning is a new study way developed in recent years the theory and technology are not mature enough. But these beneficial attempts for mobile learning opens up new learning model. The future society is a society full of mobile information. Although the overall mobile education is premature, but the continuous development of communication technology, intelligent mobile phone performance improvement and falling prices, and education, life-long education continued demand, will certainly bring mobile education wide development prospects. According to the system requirements, the system uses C/S architecture is shown in figure 1. Client systems through Web services access to course learning resources and related information. The Web service receives a client request, access the database to acquiring resources and information, and the results back to the client. Server management system is responsible for managing the users of the system, student information, course information, curriculum resources and learning process data. Manufacturer of smart phones are different, the operating system they choose is very different and therefore the application of the software is essentially incompatible with each other. Nokia and MOTOROLA, for example: Nokia is using Symbian S60 system, it is a pure 32-bit, real-time multitasking operating system, has the characteristics of low power consumption, less memory footprint, very suitable for used in mobile devices such as mobile phones; While MOTOROLA company is using a Linux system, this is an open source mobile operating platform, the platform by the operating system, middleware, user 
interface and application software of mobile terminal claims to be the first to make truly open and complete mobile software. The two companies using the operating system of far, each company has no ability refers to the use of the operating system and become a unified standard, creates in such intangible resources can make full use of, for mobile learning promotion and the emperor an invisible system. According to the system requirements, the server-side data management system is responsible for the server maintenance operation such as add, modify, delete, and query.

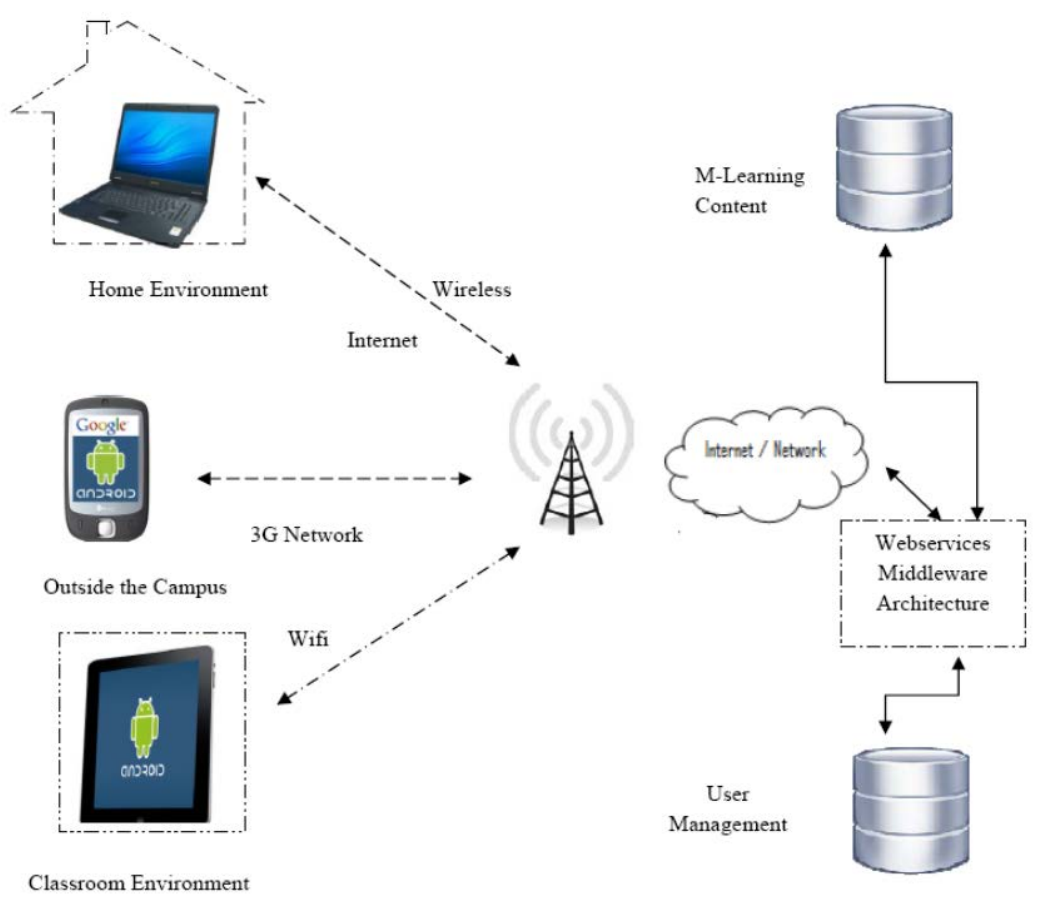

Figure 1.The General Structure of the Proposed Framework

The General Design of the Framework for Web Port. Collaborative learning is based on that learning is a natural social behavior of learners to speak. Every day is more and more important in all education environment is implanted stage, from primary school to university. Mobile equipment with advanced wireless technology to our education learning at all levels of level. Mobile technology has been adapted as the main communication, because it is very suitable for collaborative learning environment. The concept of mobile collaborative learning from the classroom - (production line) -based learning is totally different. The teaching method of learning offers many possibilities, such as a group of people the opportunity, the work done in the same or different organization to participate in specific target using mobile devices. The mobile learning application is a software product for mobile devices. To access lectures on an individual student mobile device, the required application should be downloaded from their organization website or other places. The application provides a great mobile- based platform for learners to use the learning system and to develop templates that would be useful in the workplace. Once fully download, it must be installed to their mobile device. Application on the mobile phone screen display icon indicates a process is visiting members of the group or class. Learning application should also include the media player supports audio and video files, open lecture material and uninterrupted. The initial display courses should contain the title and abstract, student registration. Smartphone replacement fast, but rapid development at the same time inevitably lead to the instability of the foundation of the this problem cause many manufacturers put attention on the upgrading of mobile phone hardware, software, and on the smartphone appeared "small problems" turn a blind eye. Such as mobile phone battery capacity is small, for a long time the Internet hardware consumption caused by triggering network signal is not stable, slow boot reaction, etc. MCL to become a successful learning application, basic operation tools support should include the following options: visual process of cooperation, discussion process, access to the user and other mobile learning options such as class lecture, test, interactive quizzes, grades and so on. In addition, shaping a culture sensitive learning experience, the application will need to multimedia network 
environment. Finally, it is very important to carefully designed study will be developed application combined with middleware architecture configuration support. The web port is shown in the figure 2 .

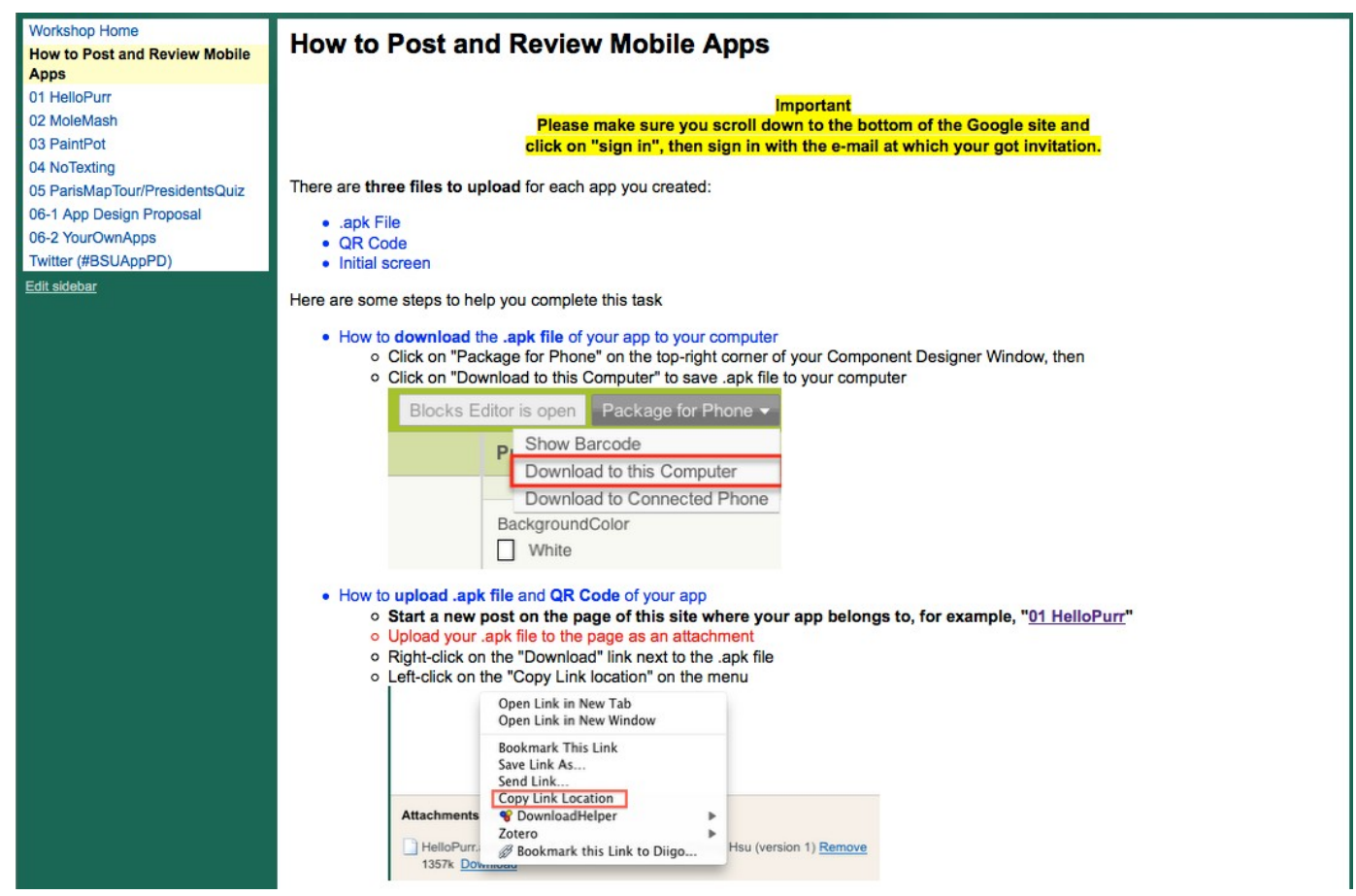

Figure 2.The UI Design of the Web Port

The General Design for the Mobile Port. Production line application is a separate application, use and termination of service, they no longer needed. It should also be designed to use Windows form or Web browser application of client-server model. Android is an open source mobile operating system and application framework supported by Google. By providing an open development platform, Android offers developers to build extremely rich and innovative applications with a rich set of user interface. Provide technical support which makes the mobile learning more active. Mobile learning resources are present in the form of multimedia, and a variety of media formats the intelligent mobile phone technology development make mobile learning has been the biggest technical support. The user interface for the mobile port is shown in the figure 3.
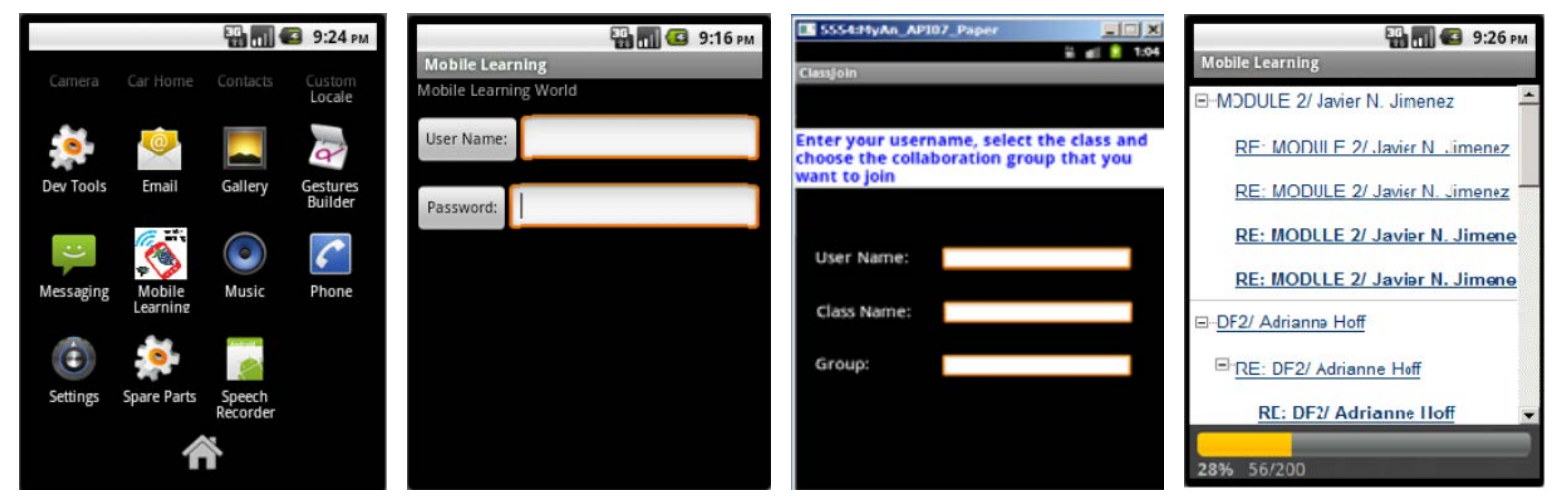

Figure 3.The UI Design of the Mobile Port

Once students have entered the registered class, whether they received survey involved in cooperative learning process depends on the kind of demand. MCL prototype is designed for users to easily work with mobile. Generally speaking, it needs enterprise database warehouse (EDW) to help students find and search the necessary information when you log in to a class or content. Initially, the enterprise data warehouse provides standard teaching material information, course information, including name, ID, course description, discussed topics, and so on. In a well-organized collaborative learning environment, it is very important to set the appropriate team members, set up a BBS based on 
the research topic. BBS of the communication is necessary to build an organization more parties and affinity, and provide a simple way to set a goal, find the same goal.

\section{Experiment and Simulation Result}

MCL should connect with online classes, in which way, students can go out for accessing and completing the class room upon their request. In MCL, online classes should include three types of windows, namely media windows, lecture window, and class network window. The following figure shows the actual appearance of the designed system. Users after a successful login into the system main interface, as shown, can choose to view the course information in the system menu, enter the course learning, online update or load the local resources, etc. When using local resources update methods, learning resources should be in the heart of the PC file downloaded to memory card, then the system main interface, select the "resource local update" command to complete resources updates. The Activity call ClientRequest and myHandler object submit a service request to the server, request access to the results. Activity is implemented in the Android system function of the main tool and users can quickly complete the system design, and through a variety of UI component interface design, greatly improve the interactivity of the system.

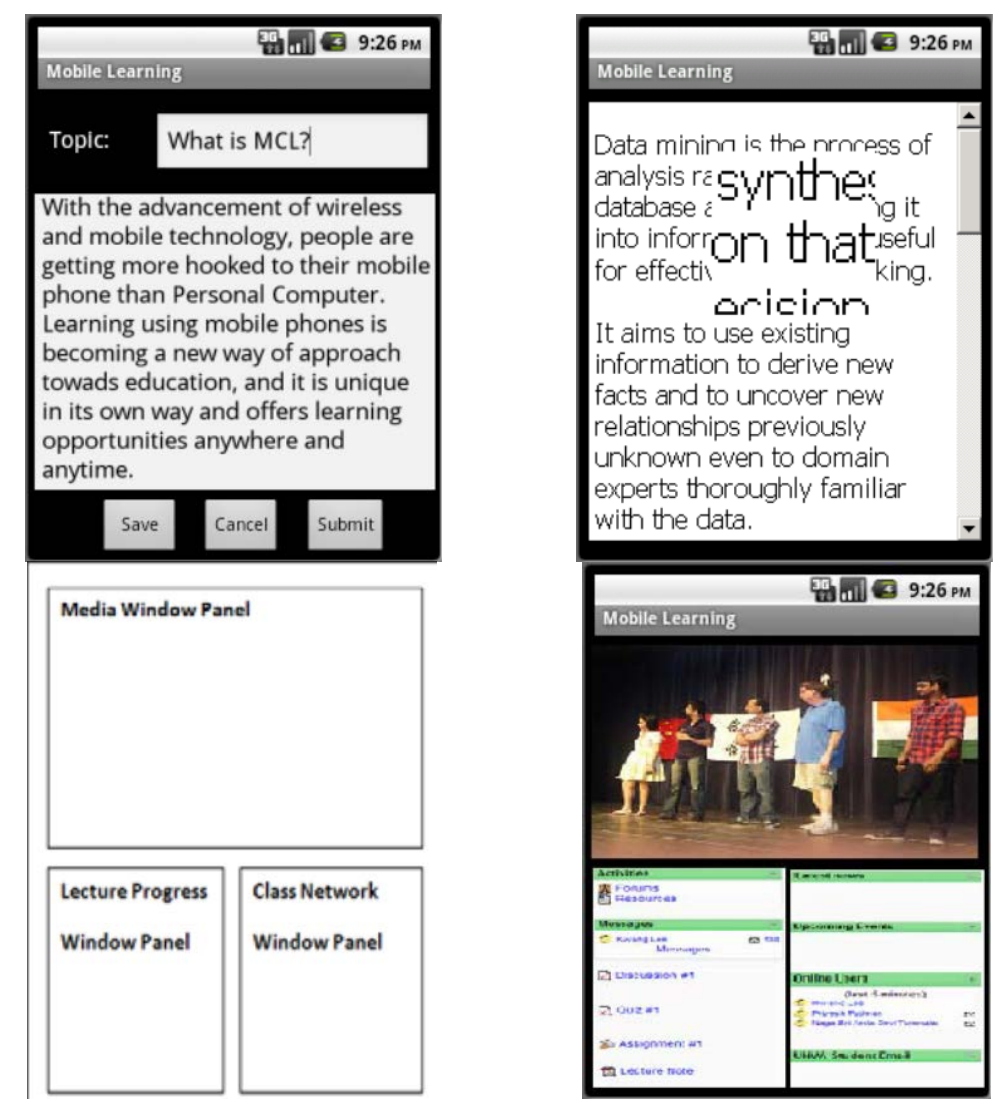

Figure 4.The Execution of the System

\section{Conclusion and Summary}

In view of the existing mobile learning system interactive poor, renewable resources, shortcomings and so on big data traffic, this paper puts forward a kind of mobile learning system based on Android platform. The system can not only solve the above problem, also can easily implement integration and extension. With the rapid popularization of smart mobile terminals, mobile study become a hot spot, the trend of traditional live remote education system puts forward a new challenge. Future smartphone gradually to the diversification, human development, especially the $3 \mathrm{~d}$ in the mobile phone is gradually developed, which has injected new vitality to the development of smart phones. And the service life of the battery, the security of the mobile phone and GPS functions such as 
research and development and to solve also makes the smartphone brought off for mobile learning basis, as for whether to use smart phones for mobile learning, it also requires us to change the idea, set up the correct learning concept, do it anytime, anywhere learning, this is the use of mobile phones for the future study of our expectations. This article in view of the existing mobile learning system of poor interactivity, renewable resources, and the disadvantages, with big data traffic based on Android platform of mobile learning system is designed. The system has good interactivity, convenient resource updates, and the main characteristics of small data traffic. Based on Android platform of mobile learning system can be widely used in mobile phone, PDA, tablet or other portable, service for the learning resources, provide a new way to research on mobile learning system has certain practical and reference value. In the future, we plan to modify the current user interface to polish the system.

\section{References}

[1] Alam, F., Panda, P. R., Tripathi, N., \& Sharma, N. (2014). Energy optimization in Android applications through wakelock placement. Design, Automation and Test in Europe Conference and Exhibition (DATE), 2014 (pp.1 - 4). IEEE.

[2] Afonso, V. M., Amorim, M. F. D., Grégio, A. R. A., Junquera, G. B., \& Geus, P. L. D. (2014). Identifying android malware using dynamically obtained features. Journal of Computer Virology and Hacking Techniques.

[3] Li, D., Hao, S., Gui, J., \& Halfond, W. G. J. (2014). An Empirical Study of the Energy Consumption of Android Applications. Software Maintenance and Evolution (ICSME), 2014 IEEE International Conference on (pp.121 - 130). IEEE.

[4] Sendra, S., Granell, E., Lloret, J., \& Rodrigues, J. J. (2014). Smart collaborative mobile system for taking care of disabled and elderly people. Mobile Networks and Applications, 19, 3, 287-302.

[5] Couto, M., Carção, T., Cunha, J., Fernandes, J. P., \& Saraiva, J. (2014). Detecting anomalous energy consumption in android applications. Lecture Notes in Computer Science.

[6] *, Q. C., Lai, H., Ge, W. Q., \& Qi, M. J. (2014). Research and development of mobile termination for the steel quality evaluation system based on android. Applied Science, Materials Science and Information Technologies in Industry.

[7] Zhao, Z., Ma, L., Cheung, Y., Wu, X., Tang, Y., \& Chen, C. L. P. (2015). Apleaf: an efficient android-based plant leaf identification system. Neurocomputing, 151, 1112-1119.

[8] Kilinc, C., Mostafa, S. A. M., Islam, R. U., Shahzad, K., \& Andersson, K. (2014). Indoor Taxi-Cab: Real-Time Indoor Positioning and Location-Based Services with Ekahau and Android OS. Innovative Mobile and Internet Services in Ubiquitous Computing (IMIS), 2014 Eighth International Conference on (pp.223 - 228). IEEE. 